\title{
Translation and psychometric testing of the Icelandic version of the MISSCARE Survey
}

\author{
Helga Bragadóttir RN, PhD (Associate Professor, Chair Nursing Administration) ${ }^{1,2}$, Beatrice J. Kalisch RN, PhD, \\ FAAN (Titus Professor) ${ }^{3}$, Sigríður Bríet Smáradóttir Cand. Psych. (Research Assistant) ${ }^{1}$ and Heiður Hrund
} Jónsdóttir MSc (Statistical Consultant) ${ }^{4}$

${ }^{1}$ University of Iceland Faculty of Nursing, School of Health Sciences, Reykjavik, Iceland, ${ }^{2}$ Landspitali University Hospital, Reykjavik, Iceland, ${ }^{3}$ University of Michigan, School of Nursing, Ann Arbor, MI, USA and ${ }^{4}$ The Social Scinence Research Institute, University of Iceland, Reykjavík, Iceland

Scand J Caring Sci; 2015; 29; 563- 572

Translation and psychometric testing of the Icelandic version of the MISSCARE Survey

Background: Missed nursing care, required standard care that is not provided, is a relatively new concept in nursing, and prior to this study, it had not been discussed in Iceland.

Aim: To successfully translate the MISSCARE Survey from US English to Icelandic.

Method: The translation and psychometric testing of the MISSCARE Survey-Icelandic was completed in six steps: (1) forward translation, (2) revision of the translation, (3) back-translation, (4) revision of the back-translation, (5) pilot-testing, (6) data collection and psychometric testing. Back-translation included work of linguists, clinicians and scholars in the original and target country. Psychometric testing was completed on data from a pilot-test and a national study. The target population was nursing staff providing patient care in medical, surgical and intensive care units in hospitals in Iceland. Pilot study data were collected in November-December 2011, and data for the national study were collected in March-April
2012. The MISSCARE Survey asks about missed nursing care activities (part A), and reasons for missed nursing care (part B), besides demographic and background questions.

Results: Response rate for the pilot study was 57\% (67/ $118)$, and for the national study, it was 69\% (599/864) with good acceptability. Overall test-retest Pearson's correlation coefficient for part A was 0.782 ( $p<0.001)$ and $0.530(p<0.05)$ for part B. Cronbach's alpha reliability coefficient for the overall part B and subscales ranged from 0.795-0.894. Confirmatory factor analysis for part B indicated a good model fit to the three factors: Communication, Material resources and Labour resources.

Conclusion: The MISSCARE Survey was successfully translated from US English to Icelandic, using a stringent back-translation method. The Icelandic version tested reliable and valid. This study supports global use of the MISSCARE Survey.

Keywords: missed nursing care, hospitals, translations, psychometrics.

Submitted 3 January 2014, Accepted 24 April 2014

\section{Introduction}

For the purpose of surveying missed nursing care in Icelandic hospitals, the MISSCARE Survey was translated from US English to Icelandic and tested. A four-step backtranslation method was employed along with a two-step testing for its psychometric properties. Previous studies indicate that even though translation is being done between related languages from countries with somewhat

Correspondence to:

Helga Bragadóttir, University of Iceland Faculty of Nursing, School of Health Sciences, Eirberg, Eiriksgata 19, 101 Reykjavik, Iceland.

E-mail: helgabra@hi.is comparable cultures, the translation needs to capture both the content and the meaning in the questionnaire, referring to cultural adaptation of the new version (1-7). Language and culture play the key roles as comparable words or concepts may not be found in the different languages or that literally translated words may have different cultural meanings between languages or countries. The final steps for instrument translation are to identify the reliability and validity of the instrument in the target language with the target population. Psychometric testing of translated instruments is essential for valid and reliable data collection and for cross-cultural comparison.

Missed nursing care being a relatively new concept in nursing, Icelandic nurses were not acquainted with it or 
its meaning. As a defined phenomenon, 'missed nursing care' did not exist in the Icelandic language nor was it used to describe errors of omission in nursing care in Iceland. This brought several challenges to the work of translating the MISSCARE Survey into Icelandic, challenges which fall under what Brislin (8) calls emic-etic distinction, emic analysis referring to the values of each culture under study, etic analysis referring to being able to make cross-cultural generalisations. The purpose of this paper is to describe the translation of the MISSCARE Survey from US English to Icelandic and the results of psychometric testing.

\section{Background}

The MISSCARE Survey was developed and tested in the United States in a rigorous five-phase process including both qualitative and quantitative methods (9). Missed nursing care was first identified as a phenomenon in nursing by Kalisch (10) in a focus group study. A total of 107 Registered Nurses (RNs), 15 licensed practical nurses (LPNs) and 51 nursing assistants (NAs) in medical-surgical units were interviewed using a semi-structured interview design asking about nursing care regularly missed on their unit and reasons for not completing this care. Study findings revealed nine themes of regularly missed nursing care and seven themes of reasons for routinely missing nursing care. The missed nursing care themes identified were ambulation, turning, feeding, patient teaching, discharge planning, emotional support, hygiene, intake and output documentation, and surveillance. Identified reasons for routinely missing nursing care were as follows: too few staff, time required for the nursing intervention, poor use of existing staff resources and 'it's not my job' syndrome (10). Following the focus group study (10), the concept of missed nursing care was analysed and presented using a stringent eight-step method by Kalisch et al. (11). The attribute categories identified to contribute to missed nursing care are as follows: (1) antecedents that catalyse the need to decide about priorities in regard to demand for patient care, labour and material resources, and communication; (2) elements of the nursing process including assessment, planning, interventions and evaluation; and (3) internal perceptions and values of each nurse and the team (11). Missed nursing care is defined as an error of omission as it 'refers to any aspect of required care that is omitted either in part or in whole or delayed' $(9$, p. 211). Missed nursing care is assumed to be directly related to patient outcomes (11).

Based on study findings about missed nursing care, a middle range explanatory theory on The Missed Nursing Care Model was developed followed by the development of a quantitative questionnaire, the MISSCARE Survey (9, $12,13)$. Items of measure were identified from qualitative study findings, concept analysis, interviews with key informants and pilot-testing (9). A content validity index of 0.89 was gained with a total of 19 staff nurses serving on one of three panels of experts. Further content validity testing was done by interviewing 95 additional nurses and testing the actual instrument on 25 nurses (9).

The MISSCARE Survey has been tested reliable and valid for adult inpatient medical, surgical and intensive care hospital units. Psychometric testing of the MISSCARE Survey was completed in two studies in the United States and included measures of acceptability, validity and reliability. Acceptability in both studies was satisfactory, as for study one, $85 \%$ of respondents answered all the questions and additional $14.1 \%$ omitted three or less questions. In study two, $92.6 \%$ answered all the questions. The questionnaire has two parts on missed nursing care, part A on nursing care activities and part B on reasons for missed nursing care. Exploratory factor analysis (EFA) for part A did not reveal consistent factor loading indicating the items to be independent from each other. One item (attending interdisciplinary care conferences) was eliminated from part A between study one and study two as few participants indicated that item to be missed (9). However, two items (attending interdisciplinary care conferences and wound care) were added to part $\mathrm{A}$ in a later version of the questionnaire, leaving it with 24 items (12). For part B, a three-factor solution was confirmed with confirmatory factor analysis (CFA) in study one and study two: communication, labour resources and material resources. In study one, the three-factor solution accounted for $51.13 \%$ of the variance and in study two for $52.25 \%$. The 16 items in part B all loaded greater than 0.35 on one of the three factors (9). Later, the 17th item (heavy admissions and discharge activity) was added to part B of the MISSCARE Survey (12).

The MISSCARE Survey has been translated to several languages, besides Icelandic, and is being tested for reliability and validity. The Turkish version of the survey, the MISSCARE Survey-Turkish, tested both reliable and valid with a sample of 436 staff nurses in four acute care hospitals in Turkey, confirming previous results in the United States (14). A Portuguese version of the questionnaire is being tested and validated for use in Brazil, with satisfactory results from a pretest with 60 nursing staff from one university hospital (15).

\section{Methods}

The translation and psychometric testing of the MISSCARE Survey-Icelandic was completed in six steps, displayed in Fig. 1. Steps 1-4 include the back-translation process.

\section{Participants}

Participants answering the MISSCARE Survey-Icelandic were Registered Nurses (RNs), practical nurses (PNs), 


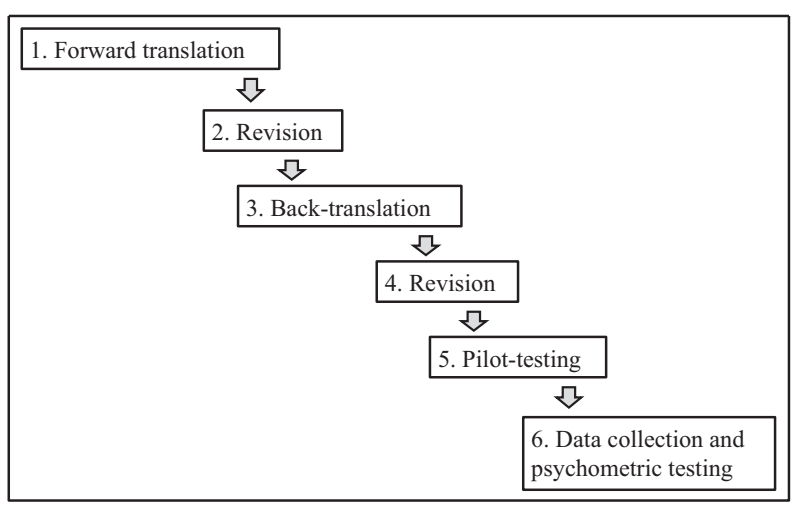

Figure 1 The back-translation process.

assistive personnel providing direct patient care, nurse managers and assistant managers. Nursing care in Icelandic hospitals is almost entirely carried out by RNs and PNs. In Iceland, $70 \%$ of RNs have at least a 4 -year baccalaureate degree in nursing (personal information from the Icelandic Nurses Association, June 12 2013). Most PNs have a 3-year vocational level education. PNs are defined as nursing assistive personnel working under the supervision of RNs in hospitals. In Iceland, RNs and PNs are licensed healthcare professionals. Health care in Iceland is nationalised, and all the participating hospitals are governmentally run.

\section{The MISSCARE Survey}

The version of the MISSCARE Survey used in this study is the most recent one, published by Kalisch in 2009 (12). The MISSCARE Survey has three parts: (1) questions on demographic and background variables; (2) questions on nursing care activities (part A on missed nursing care); (3) questions about the reasons for omitting or delaying nursing care activities (part B on missed nursing care). All questions on demographic and background variables are multiple choice questions except one asking about the number of patients cared for during present or last shift. Part A consists of 24 items. On a 5-point Likerttype scale ranging from 'always missed' to 'never missed', participants are asked to rate how frequently each element is missed by the nursing staff on their unit. Part B consists of 17 items. On a 4-point Likert-type scale ranging from 'significant reason' to 'not a reason for missed nursing care', participants indicate the reasons nursing care is missed on their unit.

\section{The back-translation process}

A type of the back-translation method derived from Brislin $(1,8,16)$ and Carlson (17) was utilised using similar methods described by a number of other scholars (2-5).
The importance of the two main parts of translation, language and content, was emphasised.

Iceland is a European Nordic country; however, its location between the continents of America and Europe gives it cultural exposure to both these neighbouring continents. Icelanders are about 320.000 and speak their own language, Icelandic. Icelandic is related to English in the way that it is the Old Norse language of Germanic origin.

The forward translation included separate first translation from US English to Icelandic by two bilingual nurses with doctoral preparation, working in academia. These two nurses then combined their translations into one Icelandic version.

The second step was to evaluate and revise the translation of the Icelandic translation. This was done by one PhD-prepared nurse working in academia and an experienced elementary school teacher with a master of public health degree. Neither of these evaluators had seen the original version of the survey. Their comments and suggestions, which were minor, were then taken into consideration. Following their revision, a professional translator evaluated and compared the Icelandic translation to the US English version. Slight changes were made to the translation of the survey.

The back-translation was done by a professional translator who had not seen the original US version of the survey. The revision of the back-translation included a comparison of the original US version of the survey to the back-translated version, by three doctoral students in nursing in the United States. They rated each paragraph and item on a three-point scale as to whether the wording and content were exactly the same, whether the wording was different but not the content or whether the wording and the content were different. The majority of the back-translated version was evaluated as having the exact wording and content, and no text or item was rated as different in terms of wording and content. The wording evaluated as different was revised by the two bilingual PhD-prepared nurses who did the forward translation. Following this revision of the Icelandic version of the survey, it was proofread by an Icelandic linguist. The revised MISSCARE Survey-Icelandic was then evaluated by two newly graduated Registered Nurses (RNs) who separately answered the questionnaire. These RNs were well acquainted with publications on previous studies on missed nursing care in the United States. They met with one of the investigators for discussion on the wording, understandability, interpretation and cultural relevance of the questionnaire. In general, they understood the survey well, however, made suggestions to changing the translation of the main concept of missed nursing care, and the wording of a few items. The final review of the Icelandic version before pilot-testing was completed by two of the investigators. 


\section{Pilot-testing}

The MISSCARE Survey-Icelandic pilot-testing was completed in November-December 2011. Participants were all nursing staff from five inpatient units at the university hospital in Iceland: one gynaecology unit, one paediatric unit and three geriatric units. The reason for not using medical, surgical and intensive care units in the pilot-testing was to keep them unexposed to the survey since they would be included in the national study. The total sample for the pilot-testing was 118 nursing staff members. A liaison person in each unit was responsible for distributing the questionnaires to all nursing staff on their unit. Enclosed with the questionnaire was an information letter, an informed consent for participants to sign and two marked envelopes for the questionnaire and the informed consent. Two weeks later (time 2), the questionnaire was sent again to those who had answered it the first time (time 1). Reminders were sent out via e-mail to nurse managers and the liaison persons who distributed them to all participants. As an incentive for participation, one name of a nursing staff member answering at time 1 and one answering at time 2, were drawn from a hat, and these participants received a gift certificate to a shopping mall in the area. Following the data collection, all units and all unit liaisons received a thank-you letter and a box of chocolate from the investigators.

\section{The testing of the national study data}

The sample for the national survey on missed nursing care consisted of 864 nursing staff in all 27 medical, surgical and intensive care units in the country. All were inpatient units, 11 of them medical, eight surgical, five medical and surgical and three intensive care units. Participating units at the university hospital were 17 (nine medical, six surgical and two intensive care) and 1-3 from each of the other seven hospitals located in different parts of the country.

Data collection was completed in March-April 2012. Unit liaison persons distributed the questionnaires to all nursing staff in their unit, with an invitation letter and a response envelope. Letters to encourage participation were sent to nurse managers of the participating units and the liaison persons. In the invitation letter, it was announced that all units who participated at or above the $50 \%$ level would receive a box of chocolates. Twenty-five out of 27 units reached this goal. All units received a thank-you letter following data collection, and all liaison persons also were sent a thank-you letter with a small token of appreciation (a key chain).

\section{Data analysis}

For the psychometric testing of the questionnaire, participants were included if they said they spent most of their working time on the unit and answered at least $70 \%$ of the items in the relevant part, A or B. Acceptability indicating ease of use (18), measured by frequency of missing data (5), was evaluated for both parts (A and B) of the MISSCARE Survey-Icelandic questionnaire with the pilot study data and the national study data. In the United States, it took staff nurses no more than 10 minutes to complete the questionnaire (9). The time it took participants in Iceland to complete their answers was not measured. For part B, Cronbach's alpha coefficient was calculated for reliability in the pilot study and the national study, and CFA for construct validity testing with the national study data. A theory-driven approach based on former studies with the MISSCARE Survey (9, 12) guided the use of CFA for part B. When confirming a theory reflected in a detailed and identified model as was the case in this study, CFA is recommended (19-21). A good fit is indicated by CFI $>0.95$, RMSEA $<0.10$ and SRMR $<0.08$ (22). The pilot-test included test-retest reliability testing using Pearson's correlation coefficient analysis for part A and part B. As reported in Kalisch and Williams (9), part A of the MISSCARE Survey contains a list of nursing actions, which are not necessarily related to one another (i.e. a nurse may not give a bath but may ambulate a patient). Therefore, neither Cronbach's alpha reliability testing nor factor analysis was appropriate for the testing of part A.

All statistical calculations were completed in IBM SPSS 20 (IBM, Armonk, NY, USA), except the CFA which utilised LISREL 8.8 (Karl Jöreskog and Dag Sörbom, Uppsala University, Uppsala, Sweden) for the calculations.

\section{Ethical considerations}

Prior to data collection, the study was approved by each hospital Institutional Review Board, or analogue body in the smaller hospitals, and the Data Protection Authorities of Iceland (S5388/2011). The participants in the pilot-test gave their written informed consent prior to participation as their names were needed for the retest. In the national survey, participation equalled a written informed consent.

\section{Results}

\section{Pilot-testing results}

The total response rate in the pilot-test was 57\% (67/ 118). The characteristics of the participants in the pilottesting are shown in Table 1. Almost all participants were females $(n=66)$, and the vast majority were RNs $(58 \%)$ and PNs $(27 \%)$. Most worked 30 hours or more each week $(73 \%)$, the majority (88\%) were 35-64 year old, $69 \%$ had 10 years or more experience in their role, and 
Table 1 The characteristics of participants in the pilot-testing $(\mathrm{N}=67)$ and the national study ( $\mathrm{N}=599$ )

\begin{tabular}{|c|c|c|}
\hline & $\begin{array}{l}\text { Pilot-testing } \\
\%\end{array}$ & $\begin{array}{l}\text { National study } \\
\%\end{array}$ \\
\hline \multicolumn{3}{|l|}{ Age } \\
\hline $26-34$ & 10 & 27 \\
\hline $35-44$ & 24 & 25 \\
\hline $45-54$ & 30 & 29 \\
\hline $55-64$ & 34 & 18 \\
\hline$\geq 65$ & 2 & 1 \\
\hline \multicolumn{3}{|l|}{ Role } \\
\hline Registered nurse (RN) & 58 & 58 \\
\hline Practical nurse (PN) & 27 & 37 \\
\hline Nursing assistant & 3 & $<1$ \\
\hline $\begin{array}{l}\text { Nurse manager / } \\
\text { assistant manager }\end{array}$ & 12 & 4 \\
\hline Other & & 1 \\
\hline \multicolumn{3}{|l|}{ Highest educational degree } \\
\hline PN Diploma & 29 & 37 \\
\hline RN Diploma & 20 & 9 \\
\hline $\begin{array}{l}\text { Bachelor's degree } \\
\text { in nursing }\end{array}$ & 48 & 50 \\
\hline $\begin{array}{l}\text { Master's degree or } \\
\text { higher in nursing }\end{array}$ & 3 & 3 \\
\hline $\begin{array}{l}\text { Master's degree or } \\
\text { higher outside of nursing }\end{array}$ & - & 1 \\
\hline \multicolumn{3}{|l|}{ Experience in role } \\
\hline Up to 6 months & 1 & 1 \\
\hline Greater than 6 months to 2 years & - & 12 \\
\hline Greater than 2 years to 5 years & 6 & 15 \\
\hline Greater than 5 years to 10 years & 24 & 17 \\
\hline Greater than 10 years & 69 & 55 \\
\hline \multicolumn{3}{|l|}{ Experience on current unit } \\
\hline Up to 6 months & 9 & 5 \\
\hline Greater than 6 months to 2 years & 8 & 17 \\
\hline Greater than 2 years to 5 years & 28 & 22 \\
\hline Greater than 5 years to 10 years & 28 & 19 \\
\hline Greater than 10 years & 27 & 37 \\
\hline \multicolumn{3}{|l|}{ Work hours } \\
\hline Days & 15 & 8 \\
\hline Evenings & 8 & 4 \\
\hline Nights & 4 & 4 \\
\hline Rotating shifts & 73 & 84 \\
\hline \multicolumn{3}{|l|}{ Unit type } \\
\hline Pediatric & 24 & \\
\hline Gynecology & 21 & \\
\hline Geriatric & 55 & \\
\hline Medical & & 35 \\
\hline Surgical & & 31 \\
\hline Mixed medical-surgical & & 17 \\
\hline Intensitve care & & 17 \\
\hline
\end{tabular}

$55 \%$ had greater than 5 years' experience on their current unit.

Just over $70 \%$ of the 67 that answered the pilot survey for missed nursing care at time 1 also answered the survey at time 2 , or 47 respondents. Out of the 47 that answered at both survey times, 26 were RNs, 14 were PNs, 5 were nurses in managerial roles, and two were nursing assistants.

Acceptability. For part A (elements of missed care) at time 1, 56 respondents indicated to spend most of their working time on the unit and answered at least $70 \%$ of the items in part A (had missing data on less than seven items out of 24) out of which $73 \%$ completed part A without omitting any item. Table 2 displays acceptability for part A and part B for all times of measures. The range of missing items in part A per participant was 0-6. Eight out of the 47 that responded at both time 1 and time 2 were removed from the data for time 2 , because they did not spend most of their time on the unit and answered less than $70 \%$ of the items. Remaining for time 2, were 39 respondents out of which $74 \%$ completed part A without omitting any item.

For part B (reasons for missed care) at time 1, 51 participants indicated to spend the majority of their time on the unit and answered at least $70 \%$ of the items (had missing data on less than five items out of 17). From these, $78 \%$ completed part B without omitting any items. The range of missing items per participant was $0-3$. At time 2, from the 37 respondents who spent most of their time on the unit and answered at least $70 \%$ of the items in part B, 97\% completed part B without omitting any item.

Reliability. Test-retest reliability for part A was completed with 39 participants that answered at time 1 and time 2 . Out of the 24 items, $59 \%$ of them were answered identically at time 1 and time 2 and $90 \%$ chose the exact

Table 2 Acceptability of the MISSCARE Survey-Icelandic

\begin{tabular}{|c|c|c|c|c|}
\hline & \multicolumn{2}{|c|}{ Part A } & \multicolumn{2}{|c|}{ Part B } \\
\hline & $N$ & $\%$ & $\mathrm{~N}$ & $\%$ \\
\hline Pilot-test time 1 & 56 & & 51 & \\
\hline No omitted item & & 73 & & 78 \\
\hline 1 Omitted item & & 9 & & 18 \\
\hline 2 Omitted items & & 4 & & 2 \\
\hline$>2$ Omitted items & & 14 & & 2 \\
\hline Pilot-test time 2 & 39 & & 37 & \\
\hline No omitted item & & 74 & & 97 \\
\hline 1 Omitted item & & 8 & & 3 \\
\hline 2 Omitted items & & 8 & & \\
\hline$>2$ Omitted items & & 10 & & \\
\hline National study & 559 & & 546 & \\
\hline No omitted item & & 78 & & 86 \\
\hline 1 Omitted item & & 11 & & 8 \\
\hline 2 Omitted items & & 3 & & 2 \\
\hline$>2$ Omitted items & & 7 & & 4 \\
\hline
\end{tabular}


same answer or the next closest answer at time 2. Simple additive scores were computed for missed nursing care, and the overall test-retest Pearson's correlation coefficient for the 24 items was $0.782(p<0.001)$.

Test-retest reliability for part $\mathrm{B}$ was completed with the 37 participants that answered at time 1 and time 2, spent most of their working time on the unit and answered $70 \%$ or more of the items at each time. Out of the 17 items, $55 \%$ of them were answered identically at time 1 and time 2 and $97 \%$ chose the exact same answer or the next closest answer at time 2 . The overall test-retest Pearson's correlation coefficient for the 17 items was $0.530(\mathrm{p}<0.05)$, and the three subscales had a test-retest coefficient ranging from 0.437 to 0.600 ( $\mathrm{p}<0.01$ ).

Internal consistency of part B calculated with Cronbach's alpha coefficient was 0.850 for time 1 and 0.894 for time 2. The subscales for part B ranged from 0.807 to 0.845 for time 1 and from 0.829 to 0.845 for time 2 .

Following the pilot-testing, minor changes were made to the wording or text of a few questions and the directions. These changes were, for example, adding directions on choosing only one answer to each question, underlining a word or making it bold to place emphasis on it and catch the attention of participants and changing the form for marking answers from grid to boxes. Figure 2 shows an example of the difference in the US format (which was the same in the pilot-test in Iceland) and the format of the questionnaire in the national study.

\section{National study results}

Overall response rate for the total sample was 69\% (599/ 864) ranging from 37 to $100 \%$ for each unit. The characteristics of the participants in the national study are shown in Table 1. Most of the respondents, or $58 \%$, were RNs $(\mathrm{n}=344), \quad 37 \%$ were PNs $(\mathrm{n}=221), 21$ respondents were nurse managers or assistant managers, two worked as nursing assistants, and eight had other titles (e.g. RN or PN students). The majority (67\%) came from the university hospital. Almost all were females $(98 \%)$, and just over half of participants were under the age of 45 years $(52 \%)$. In terms of work hours, $75 \%$ indicated to work 30 hours or more each week and $84 \%$ worked rotating shifts. The majority had greater than 10 years' experience in their current role $(55 \%)$ and greater than 5 years' experience on current unit (56\%). Twentythree of the participants had missing data on whether they spent most of their working time on the unit or not.

Acceptability. For part A, 559 participants spent most of their working time on the unit and answered at least $70 \%$ of the items. From these, $78 \%$ completed part A without omitting any item. For part A, the number of omitted items per participant ranged from 3 to 6 . Table 2 displays acceptability for part A and part B. For part B, 546 participants were included in the analysis. From these, $86 \%$ completed part B without omitting any item. For part B, omitted items per participant ranged from 3 to 5 .

Validity and reliability. The three subscales for reasons for missed nursing care (part B) that emerged in the study by Kalisch and Williams (9) were used when performing the CFA. The factors are Communication, Material resources and Labour resources. The Icelandic version contains one extra item compared with the findings reported by Kalisch and Williams (9), which is heavy admission and discharge activity. The Icelandic data fit the theoretical model

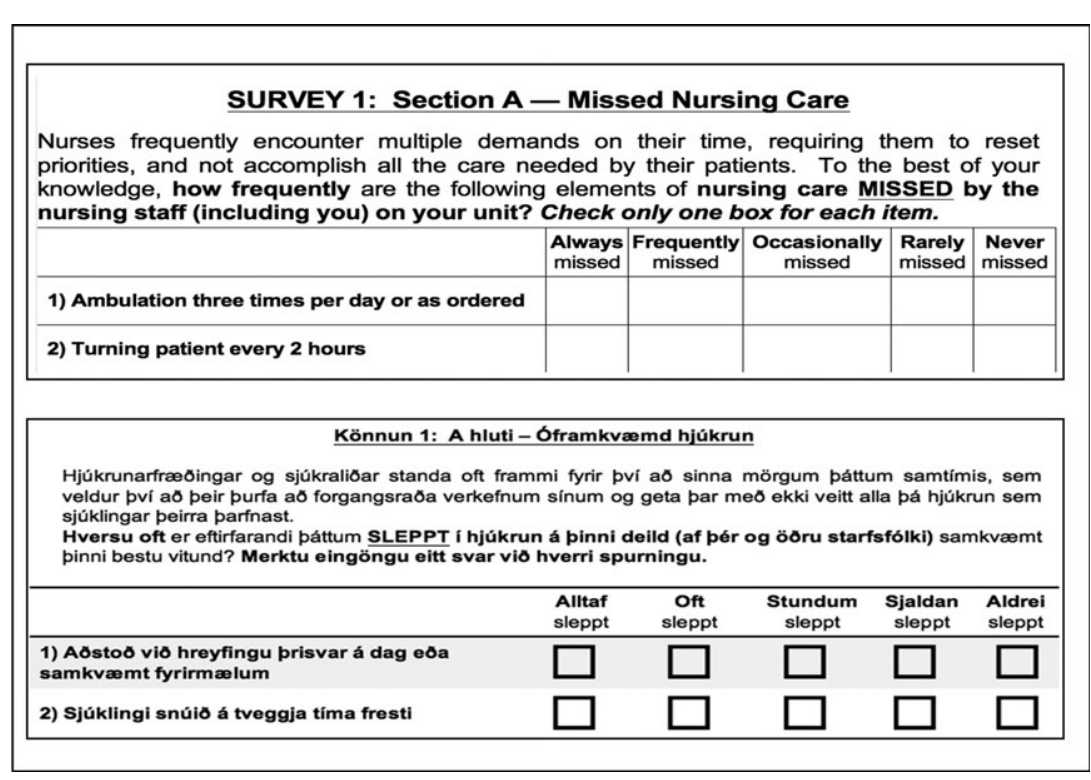

Figure 2 An example of format changes in the MISSCARE Survey-Icelandic questionnaire from the original US version to the Icelandic national study. 
with factor loadings ranging from 0.47 to 0.89 (comparative fit index $[\mathrm{CFI}]=0.971$; root-mean-square error of approximation [RMSEA] $=0.070$; incremental fit index $[\mathrm{IFI}]=0.971 ;$ standardised root-mean-square residuals $[$ SRMR $]=0.0756)$. For each subscale, the Cronbach's alpha reliability coefficient was $0.795-0.825$. Factor loadings and the Cronbach's alpha reliability coefficient for the subscales are contained in Table 3. The overall Cronbach's alpha for part B was 0.873 .

\section{Discussion}

The translation and testing of the MISSCARE Survey from US English to Icelandic was successfully carried out in six steps including a back-translation process and psychometric testing. In spite of the relationship due to a common origin of the English and Icelandic languages and Iceland being heavily exposed to US culture and language, the translation of some of the concepts and items was challenging. Especially, we found it difficult to find Icelandic terms that captured the meaning of 'missed nursing care'. This required more thorough guidance and cues to participants on how to answer the questionnaire than was provided in the original US version. Our experience can be reflected in what is referred to as cross-cultural equivalence which is important to consider when translating measures between cultures and languages (6, $7,23)$. The translation of the text, including the language and meaning, which refers to content, semantic and conceptual equivalence $(6,23)$, turned out to be more challenging than expected. The directions to participants and the interface (layout) of the questionnaire seemed to be somewhat culture-bound, referring to technical equivalence $(6,23)$. Technical equivalence has to do with the data collection method and procedure being comparable between languages and cultures when using the same tool. In our study, data collection was conducted in a comparable way as has been done in previous studies using the MISSCARE Survey questionnaire (24). However, the revision following the pilot-test led to some changes to the interface of the questionnaire. A need to secure appropriate use of the questionnaire such as participants only marking one answer to an item, and thereby strengthening the acceptability of the questionnaire, was identified. Some of the instructions were therefore set in bold letters and or by underlining words, and even adding instructions to a few variables. These changes were, however, based on subjective evaluation or face validity during the revision process following the pilot-testing and were not psychometrically tested. Our experience points out the importance of not only securing a sound back-translation of instruments in regard to language and meaning, but also taking into consideration how the visual appearance of text and format, even colours (25), may be culture-bound. The importance of a holistic, structured approach, with expert validation when translating instruments between cultures and languages, was recently highlighted in a publication on a multicountry nursing study in Europe. A questionnaire on nurses work environment was translated from US English to eleven languages in twelve countries, some of the countries having more than one language. This turned out to be a challenging process in regard to cultural differences between the United States and some of the countries, as well as in regard to linguistics not the least in countries who had more than one official language (7).This is an

Table 3 Confirmatory factor analysis for part B (reasons for missed nursing care) with factor loadings and Cronbach's alpha

\begin{tabular}{|c|c|c|c|c|c|}
\hline Factor & Cronbach's $\alpha$ & Item $B$ & 1 & 2 & 3 \\
\hline \multirow[t]{9}{*}{ 1. Communication } & \multirow[t]{9}{*}{0.825} & Tension or communication breakdowns within the nursing team & 0.74 & & \\
\hline & & Inadequate hand-off from previous shift or sending unit & 0.71 & & \\
\hline & & Lack of back up support from team members & 0.69 & & \\
\hline & & Tension or communication breakdowns with other support departments & 0.67 & & \\
\hline & & Other departments did not provide the care needed & 0.66 & & \\
\hline & & Nursing assistant did not communicate that care was not done & 0.63 & & \\
\hline & & Unbalanced patient assignments & 0.61 & & \\
\hline & & Tension or communication breakdowns with the medical staff & 0.59 & & \\
\hline & & Care giver is off unit or unavailable & 0.57 & & \\
\hline \multirow[t]{3}{*}{ 2. Material resources } & \multirow[t]{3}{*}{0.795} & Supplies/equipment not available when needed & & 0.89 & \\
\hline & & Supplies/equipment not function properly & & 0.88 & \\
\hline & & Medications were not available when needed & & 0.65 & \\
\hline \multirow[t]{5}{*}{ 3. Labour resources } & \multirow[t]{5}{*}{0.798} & Inadequate number of assistive personnel (e.g. nursing assistants, technicians, etc.) & & & 0.86 \\
\hline & & Unexpected rise in patient volume and/or acuity on the unit & & & 0.76 \\
\hline & & Inadequate number of staff & & & 0.74 \\
\hline & & Heavy admission and discharge activity & & & 0.73 \\
\hline & & Urgent patient situations (e.g. a patient's condition worsening) & & & 0.47 \\
\hline
\end{tabular}


important but scarcely discussed issue in the healthcare literature on translation of measures. Acknowledging and addressing any challenges scholars may encounter during a translation process is necessary for successful results. A sound translation is the premise of a reliable and valid instrument, which again is essential for quality data collection. We feel we achieved this in this study.

All psychometric testing of the MISSCARE Survey-Icelandic showed that it is a reliable and valid tool. The test-retest reliability of the pilot-testing data showed a strong correlation between measures at time 1 and time 2, supporting the consistency of the Icelandic version of the questionnaire comparable to test-retests of other studies using the MISSCARE Survey $(9,14)$. For part B, internal consistency was satisfactory for the overall scale as well as the subscales, as the Cronbach's alpha reliability coefficient was well over 0.70 in the pilot-test and the national study. For the construct validity of part B, the theory-driven approach was supported by a CFA (19-21). Using the data from the national study, the CFA showed a good model fit with the three factors of reasons for missed nursing care.

The response rate in our study in Iceland was good compared with former studies on the development and translation of the MISSCARE Survey. For the English version of the MISSCARE Survey used in the United States and Lebanon, the response rate reported was $53.4 \%$ and $44.4 \%$, respectively $(9,26)$, and for the Turkish version it was $67.2 \%(14)$. Acceptability was also found to be satisfactory in the Icelandic version and comparable with Brazil using a new Portuguese version, where it was $70 \%$ (15), however, less than in the United States where it was $92.6 \%$, and in Turkey were it was $100 \%$ (9).

This study has both strengths and limitations. One of its strengths was that we followed a stringent process when translating and testing the MISSCARE Survey from US English to Icelandic. Our use of a detailed and careful application of the translation process and psychometric testing resulted in a solid instrument. The high response rate and a national sample representing the whole population of the nursing staff in medical, surgical and intensive care inpatient units in one country also strengthen this study.

The main limitations of this study are that it uses an instrument which was not developed for the target language or culture it is being used in, and the small population of Iceland. Dealing with the methodological challenges of a small population is, however, the reality for scholars in Iceland, implementing both strengths and weaknesses to their studies.

\section{Conclusions and implications for future research}

Missed nursing care, or errors of omission, is of global concern which has, just recently been identified as an extensive phenomenon in nurses work reality (13-15, 27). The pressing need for reliable and valid measures for nursing care is further supported by the fact that nursing care makes a substantial contribution to quality patient care and therefore patient safety (28-34). Clinical nurses, assistive personnel, administrators and policymakers, as well as patients, must identify and respond to the importance of quality nursing care for the benefit of patients well-being and safety in health care. Identifying and responding to missed nursing care is one effort in this attempt.

Successful translation of an instrument requires a stringent strategic back-translation process and psychometric testing. This can only be done in sufficient manner by including linguists, clinicians and scholars. The importance of using a well-established instrument for translation into other languages in other cultures is also emphasised. The MISSCARE Survey has already tested reliable and valid in a number of languages and countries, which adds to its value and makes it desirable for measuring missed nursing care. The translation of the MISSCARE Survey from US English to Icelandic was successful; however, it is a work in progress and further studies will determine the applicability and strength of the tool. The use of the MISSCARE Survey globally is supported in this study.

\section{Acknowledgements}

The authors acknowledge colleagues and students who participated in the back-translation process of this study, the liaison persons who helped with data collection and participants.

\section{Author contributions}

Beatrice J. Kalisch and Helga Bragadóttir contributed to the conception and design of the study. The research proposal and translation process was supervised by Beatrice J. Kalisch and coordinated and managed by Helga Bragadóttir. Data collection was conducted by Helga Bragadóttir and Sigríður Bríet Smáradóttir. Data analysis was primarily done by Heiður Hrund Jónsdóttir. All authors contributed to the writing of the manuscript, which was led by Helga Bragadóttir.

\section{Ethical approval}

Prior to data collection, the study was approved by each hospital Institutional Review Board (3/2012), or analogue body in the smaller hospitals, and the Data Protection Authorities of Iceland (S5388/2011). The participants in the pilot-test gave their written informed consent prior to participation and in the national survey, participation equalled a written informed consent. The participating hospitals were as follows: Landspítali Háskólasjúkrahús, 
Heilbrigðisstofnun Suðurnesja, Heilbrigðisstofnun Suðurlands, Fjórðungssjúkrahúsið á Neskaupsstað, Heilbrigðisstofnun Pingeyinga, Sjúkrahúsio á Akureyri, Fjórðungssjúkrahúsið á Ísafirði, Heilbrigðisstofnun Vesturlands.

\section{Funding}

This study was funded by research grants from Landspitali-University Hospital, the University of Iceland and The Association of Registered Nurses in Iceland.

\section{References}

1 Brislin RW. Back-translation for cross-cultural research. J Cross Cult Psychol 1970; 1: 185-216.

2 Raholm MB, Thorkildsen K, Lofmark A. Translation of the Nursing Clinical Facilitators Questionnaire (NCFQ) to Norwegian language. Nurse Educ Pract 2010; 10: 196-200.

3 Sperber AD. Translation and validation of study instruments for crosscultural research. Gastroenterology 2004; 126(1 Suppl 1): S124-8.

4 Wild D, Grove A, Martin M, Eremenco S, McElroy S, Verjee-Lorenz A, Erikson P; ISPOR Task Force for Translation and Cultural Adaptation. Principles of Good Practice for the Translation and Cultural Adaptation Process for Patient-Reported Outcomes (PRO) Measures: report of the ISPOR Task Force for Translation and Cultural Adaptation. Value Health 2005; 8: 94-104.

5 Eldh AC, Ehrenberg A, Squires JE, Estabrooks CA, Wallin L. Translating and testing the Alberta context tool for use among nurses in Swedish elder care. BMC Health Serv Res 2013; 13: 68.

6 Flaherty JA, Gaviria FM, Pathak D, Mitchell T, Wintrob R, Richman JA, Birz S. Developing instruments for cross-cultural psychiatric research. $J$ Nerv Ment Dis 1988; 176: 257-63.

7 Squires A, Aiken LH, van den Heede K, Sermeus W, Bruyneel L, Lindqvist R, Schoonhoven L, Stromseng I, Busse R, Brzostek T, Ensio A, Moreno-Casbas M, Rafferty AM, Schubert M, Zikos D, Matthews A. A systematic survey instrument translation process for multi-country, comparative health workforce studies. Int J Nurs Stud 2013; 50: 264-73.

8 Brislin RW. Comparative research methodology: cross-cultural studies. Int J Psychol 1976; 11: 215-29.

9 Kalisch BJ, Williams RA. Development and psychometric testing of a tool to measure missed nursing care. J Nurs Admin 2009; 39: 211-9.
10 Kalisch BJ. Missed nursing care: a qualitative study. J Nurs Care Qual 2006; 21: 306-13; quiz 14-5.

11 Kalisch BJ, Landstrom GL, Hinshaw AS. Missed nursing care: a concept analysis. J Adv Nurs 2009; 65: 150917.

12 Kalisch BJ. Nurse and nurse assistant perceptions of missed nursing care: what does it tell us about teamwork? J Nurs Admin 2009; 39: 485-93.

13 Kalisch BJ, Landstrom G, Williams RA. Missed nursing care: errors of omission. Nurs Outlook 2009; 57: 3-9.

14 Kalisch BJ, Terzioglu F, Duygulu S. The MISSCARE Survey-Turkish: psychometric properties and findings. Nurs Econ 2012; 30: 29-37.

15 Siqueira LD, Caliri MH, Kalisch B, Dantas RA. Cultural adaptation and internal consistency analysis of the MISSCARE Survey for use in Brazil. Rev Lat Am Enfermagem 2013; 21: 610-7.

16 Jones PS, Lee JW, Phillips LR, Zhang XE, Jaceldo KB. An adaptation of Brislin's translation model for crosscultural research. Nurs Res 2001; 50: 300-4.

17 Carlson ED. A case study in translation methodology using the healthpromotion lifestyle profile II. Public Health Nurs 2000; 17: 61-70.

18 Waltz CF, Strickland O, Lenz E. Measurement in Nursing and Health Research. 2005, Springer Publishing Company, New York.

19 Carmines EG, Zeller RA. Reliability and Validity Assessment. 1979, Sage Publications, Beverly Hills.

20 Hurley AE, Scandura TA, Schriesheim CA, Brannick MT, Seers A, Vandenberg RJ, Williams LJ. Exploratory and confirmatory factor analysis: guidelines, issues, and alternatives. J Organ Behav 1997; 18: 667-83.

21 Nunnally JC. Psychometric Theory. 1967, McGraw-Hill Book Company, New York.

22 Bartholomew DJ, Steele F, Galbraith J, Moustaki I. Analysis of Multivariate
Social Science Data, 2nd edn. 2008, Chapman and Hall/CRC, London.

23 Hilton A, Skrutkowski M. Translating instruments into other languages: development and testing processes. Cancer Nurs 2002; 25: 1-7.

24 Kalisch BJ, Lee KH. The impact of teamwork on missed nursing care. Nurs Outlook 2010; 58: 233-41.

25 Fanning E. Formatting a paper-based survey questionnaire: best practices. Pract Assess Res Eval 2005; 10: 1-14.

26 Kalisch BJ, Doumit M, Lee KH, Zein JE. Missed nursing care, level of staffing, and job satisfaction: Lebanon versus the United States. J Nurs Admin 2013; 43: 274-9.

27 Ball JE, Murrells T, Rafferty AM, Morrow E, Griffiths P. 'Care left undone' during nursing shifts: associations with workload and perceived quality of care. BMJ Qual Saf 2014; 23: 116-25.

28 Aiken LH, Sloane DM, Bruyneel L, Van den Heede K, Griffiths P, Busse R, Diomidous M, Kinnunen J, Kózka M, Lesaffre E, McHugh MD, Moreno-Casbas MT, Rafferty AM, Schwendimann R, Scott PA, Tishelman C, van Achterberg T, Sermeus W; for the RN4CAST consortium. Nurse staffing and education and hospital mortality in nine European countries: a retrospective observational study. Lancet 2014; pii: S0140-6736 (13)62631-8. doi: 10.1016/S01406736(13)62631-8. [Epub ahead of print].

29 Aiken LH, Clarke SP, Sloane DM, Lake ET, Cheney T. Effects of hospital care environment on patient mortality and nurse outcomes. $J$ Nurs Admin 2008; 38: 223-9.

30 Lucero RJ, Lake ET, Aiken LH. Nursing care quality and adverse events in US hospitals. J Clin Nurs 2010; 19: 2185-95.

31 Liu K, You LM, Chen SX, Hao YT, Zhu XW, Zhang LF, Aiken LH. The relationship between hospital work environment and nurse outcomes in Guangdong, China: a nurse 
questionnaire survey. $J$ Clin Nurs 2012; 21: 1476-85.

32 Aiken LH, Sermeus W, Van den Heede K, Sloane DM, Busse R, McKee M, Bruyneel L, Rafferty AM, Griffiths P, Moreno-Casbas MT, Tishelman C, Scott A, Brzostek T, Kinnunen J, Schwendimann R, Heinen M, Zikos D, Sjetne IS, Smith HL,
Kutney-Lee A. Patient safety, satisfaction, and quality of hospital care: cross sectional surveys of nurses and patients in 12 countries in Europe and the United States. BMJ 2012; 344: e1717.

33 Kalisch BJ, Tschannen D, Lee KH. Missed nursing care, staffing, and patient falls. J Nurs Care Qual 2012; 27: 6-12.

34 Estabrooks CA, Midodzi WK, Cummings GG, Ricker KL, Giovannetti P. The impact of hospital nursing characteristics on 30-day mortality. $J$ Nurs Admin 2011; 41(7-8 Suppl): S58-68. 